\title{
Controlling mutational chaos
}

An integrative analysis of mutations at different prostate cancer stages occurring from the genomic to the proteomic level describes the relationship of changes at different molecular levels and gives an extensive overview of the proteomic aberrations. The researchers also discovered previously uncharacterized events that might yield novel biomarkers or therapeutic targets.

"We aimed at a comprehensive dissection of the molecular changes to understand how prostate cancer forms and becomes resistant to treatments," explains Tapio Visakorpi, senior author of the study. "In the new study, we used our cohort of primary and castration-resistant prostate cancer samples, which we had previously analysed for genetic, epigenetic, and transcriptional changes, to study the changes at the protein level on a large scale."

The team analysed prostate tissue from patients with benign prostatic hyperplasia (BPH) (as a model for benign tissue), nontreated prostate cancer (nPC), and castration-resistant prostate cancer (CRPC) using unlabelled mass spectrometry. To define proteomic changes during cancer development, the team compared $\mathrm{BPH}$ samples with nPC samples, identifying differential expression of 728 proteins. To characterize cancer progression and

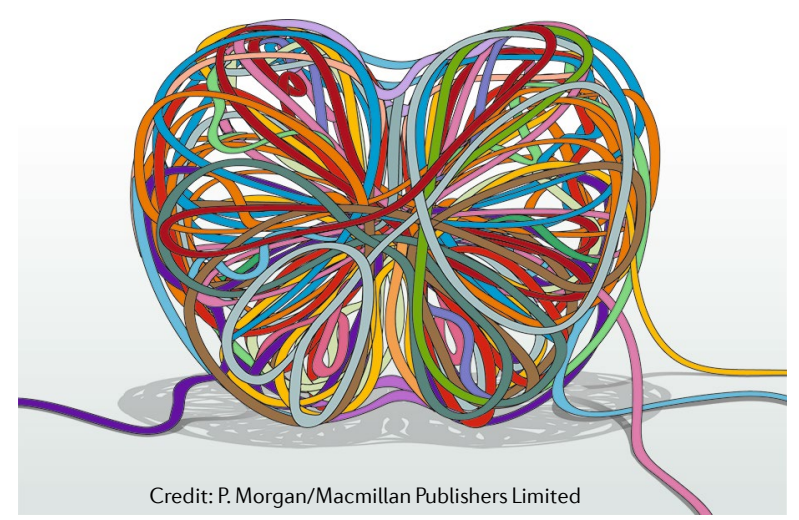

castration resistance, they compared nPC samples with CRPC samples, identifying 382 differentially expressed proteins. Overall, classes of the differentially expressed proteins were similar, but only 153 of the proteins were present in both comparison groups. Notably, expression level profiles were distinct between the sample groups, highlighting that the prostate proteomic profile undergoes considerable changes during cancer development and progression.

The team had previously performed genome, DNA methylation, and transcriptome sequencing for most of the samples. Comparison of correlations of gene copy number (GCN) and mRNA or protein levels showed that GCN and mRNA expression had a higher overall correlation in the CRPC than in the $\mathrm{nPC}$ samples, but that this difference did not occur at the protein level. Comparison of correlations with DNA methylation instead of GCN yielded similar results; but altered methylation did affect protein levels for some genes. These data indicate that genomic and epigenomic changes do not directly result in protein expression changes in prostate cancer.

The team then characterized the relationship between mRNA and protein levels. Globally, protein expression levels correlated with those of their mRNAs; however, the correlation was lower in CRPC samples than in $\mathrm{BPH}$ or $\mathrm{nPC}$ samples. Investigating whether similar genes were differentially expressed based on both mRNA and protein expression data, the team found that only few differentially expressed genes were shared between sample type pairs and that these were fewest for the nPC-CRPC comparison. Thus, transcriptomic and proteomic analyses seem to characterize different mutational events during cancer development and progression. To elucidate causes of discrepancies between the mRNA and protein data, the team analysed possible effects of microRNAs (miRNAs). Only some of the identified miRNA targets were common to both the mRNA and protein level, highlighting the importance of analysis at both levels. The data revealed several regulatory events that had not previously been identified in transcriptome data of prostate cancer samples.

"The most striking finding was how much of the genetic and transcriptomic aberrations that have accrued in advanced prostate cancer are 'buffered out' by the cancer cells," highlights Visakorpi. "This discovery advocates how cancer cells need to maintain a certain level of 'sanity' in their functions in order to survive despite the accumulating aberrations in their genome."

Finally, the team explored whether proteome analysis revealed pathway changes that had not been discovered in mRNA expression data. Overall, biological functions of the pathways most often found by transcriptomics or proteomics were similar, but several pathways were only seen for one of the domains, depending on the disease stage investigated. Notably, changes in the Krebs cycle were predominantly found at the protein level. Further testing revealed a two-step modulation of this metabolic pathway - the first during cancer development and the second during cancer progression - which seemed to be regulated by miRNAs.

"Our work indicates a limited number of targetable pathways in prostate cancer - several genetic and transcriptomic aberrations might feed into the same protein outputs," summarizes Visakorpi.

Clemens Thoma

ORIGINAL ARTICLE Latonen, L. et al. Integrative proteomics in prostate cancer uncovers robustness against genomic and transcriptomic aberrations during disease progression. Nat. Commun. 9,1176 (2018) 